\title{
Demonstrating Elusive Toroidal Dipolar Response in Metamaterials
}

\author{
V. A. Fedotov ${ }^{1}$, T. Kaelberer ${ }^{1}$, N. Papasimakis ${ }^{1}$, V. Savinov ${ }^{1}$, \\ A. V. Rogacheva ${ }^{1}$, D. P. Tsai ${ }^{2,3}$ and N. I. Zheludev ${ }^{1}$ \\ 1. Optoelectronics Research Centre and Centre for Photonic Metamaterials, University of Southampton, SO17 1BJ, UK \\ 2. Department of Physics, National Taiwan University, Taipei, 10617 Taiwan \\ 3. Instrument Technology Research Center, National Applied Research Laboratories, Hsinchu 300, Taiwan
}

Toroidal moments are fundamental electromagnetic excitations that cannot be represented in terms of the standard multipole expansion [1]. They were first considered by Zel'dovich back in 1957 [2], but only recently have become the subject of growing interest owing to their peculiar electromagnetic properties. Electromagnetic interactions with toroidal currents were predicted to disobey such widely accepted principle as the actionreaction equality. Toroidal currents can also form charge-current configurations generating vector potential fields in the absence of radiated electromagnetic waves. Although toroidal moments are held responsible for parity violation in nuclear and particle physics, no direct evidence of their importance for classical electrodynamics has been reported so far. This is because effects associated with toroidal moments in naturally available materials are extremely weak and usually masked by much stronger effects due to conventional electric and magnetic dipole and quadrupole moments.

Here we demonstrate examples of classical systems, namely metamaterials, the electromagnetic response of which can be directly related to the resonant excitation of the toroidal dipole moment. The resonant toroidal response has been observed in 'metamaterial slabs', two-dimensional arrays of artificially engineered electromagnetic scatterers (metamolecules) of toroidal symmetry (see Fig. 1A). To emphasize the toroidal response we came up with the designs of the metamolecules where both standard electric and magnetic dipole moments induced by an incident electromagnetic wave (as well as higher multipoles) are substantially suppressed, while the toroidal response is spectrally isolated and resonantly enhanced to a detectable level (see Fig. 1B).

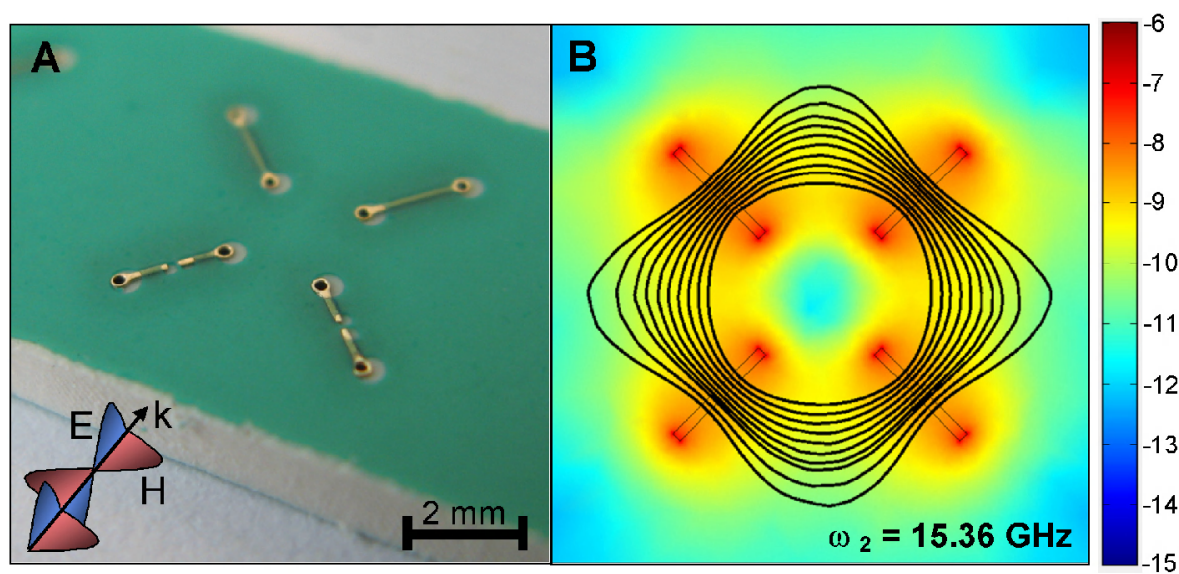

Figure 1. (A) An example of the toroidal metamolecule - an electromagnetic scatterer of toroidal symmetry composed of 4 split rings embedded vertically in a dielectric slab. (B) Toroidal dipole mode resonantly excited in the metamolecule with linearly polarized plane wave. Black lines trace magnetic field lines, while colour map shows intensity distribution of the magnetic field on a logarithmic scale.

Since toroidal excitation is routinely neglected in the constitutive relations, boundary conditions, electromagnetic forces and in the calculation of momentum loss and radiation intensity of charge-current configurations, we believe that our results indicate a need for the revision of some aspects of electrodynamics involving structures of toroidal symmetry. Furthermore, given that many biologically important molecules have elements of toroidal symmetry and may support toroidal moments such a revision could unveil new mechanism of intra-molecular interactions and genetic information transfer. Successful demonstration of the ellusive toroidal dipolar response invites us to test the most important predictions of toroidal electrodynamics, such as exotic type of optical activity, violation of Lorentz reciprocity and peculiar non-radiating systems with non-compensated vector potential [3].

\section{References}

[1] V. M. Dubovik, V. V. Tugushev "Toroid moments in electrodynamics and solid-state physics", Phys. Rep. 187, 145 (1990).

[2] Ia .B. Zel'dovich, "Electromagnetic interaction with parity violation", Sov. Phys. JETP 6, 1184 (1958).

[3] T. Kaelberer et. al, "Toroidal Dipolar Response in a Metamaterial", Science 330, 1510 (2010). 